\title{
Contextualización histórica e institucionalización académica de la psicopedagogía en Argentina
}

\section{Contexto histórico e institucionalização acadêmica da psicopedagogia na Argentina}

\section{Ana Clara Ventura*}

Instituto Rosario de Investigaciones en Ciencias de la Educación-IRICE (CONICET/UNR), Rosario, Santa Fe, Argentina

\section{Raúl Gagliardi**}

Instituto Rosario de Investigaciones en Ciencias de la Educación-IRICE (CONICET/UNR), Rosario, Santa Fe, Argentina

\section{Nora Moscoloni***}

Instituto Rosario de Investigaciones en Ciencias de la Educación-IRICE (CONICET/UNR), Rosario, Santa Fe, Argentina

\begin{abstract}
RESUMEN
En Argentina, el estudio del aprendizaje y su profesionalización revistió características controvertidas y polémicas desde la institucionalización académica de la Psicopedagogía. El objetivo de este artículo es explorar los modos en que se configuró el campo psicopedagógico y los principales antecedentes que favorecieron la aparición de la práctica psicopedagógica durante la primera mitad del siglo XX y que, asimismo, posibilitaron su inserción en el ámbito universitario en 1956. En base a la revisión efectuada, el surgimiento del Sistema Educativo, la aparición de la categoría de "fracaso escolar" abordado por la práctica de las maestras particulares y la sistematización de la carrera de Psicotecnia se constituyeron como acontecimientos significativos que impulsaron la institucionalización académica de la Psicopedagogía. Se discute la relevancia de continuar con estudios de este tipo que permitan profundizar, contextualizar y comprender las raíces históricas y socio-culturales de las controversias y debates actuales en torno a esta problemática.
\end{abstract}

Palabras clave: Aprendizaje, Argentina, Psicopedagogía.

\section{RESUMO}

Na Argentina, o estudo da aprendizagem e as características profissionais controversos e vestidos a partir da institucionalização acadêmica de Psicopedagogia. O objetivo deste trabalho é explorar as maneiras pelas quais o campo foi criado psicopedagógico e os principais elementos que favoreceram o surgimento da prática psicopedagógica na primeira metade do século XX e também permitiu a sua integração na universidade em 1956. Baseado em sua análise, o surgimento do sistema educacional, o surgimento da categoria de "fracasso escolar", proferida pela a prática individual dos professores e sistematização da raça de psicotécnicos foram estabelecidos como eventos significativos que levaram à institucionalização acadêmica da 
Psicopedagogia. A relevância de tais estudos continua a aprofundar, contextualizar e entender as raízes históricas e sócio-culturais controvérsias e debates em torno deste assunto.

Palavras-chave: Aprendizagem, Argentina, Psicopedagogía.

\section{I ntroducción}

Es reconocida la problemática de establecer fronteras profesionales precisas entre la Psicología Educacional, la Educación Especial y la Psicopedagogía en I beroamérica (BRAVO, 2009). No obstante, la literatura académica indica que cuando lo psicopedagógico es entendido como una especialidad del psicólogo en el campo del aprendizaje escolar resulta relativamente más sencillo distinguir campos de acción y modelos de intervención (HENAO LÓPEZ; RAMÍREZ NIETO; RAMÍREZ PALACIOS, 2006; GARCÍA, 2002; TELLO, 2009).

En Argentina, en cambio, se fundó el 2 de mayo de 1956 la carrera universitaria "Psicopedagogía" en la Universidad del Salvador (Buenos Aires), una institución privada de carácter confesional (MÜLLER, 2000). Cabe destacar que esta titulación se constituyó como la pionera en Sudamérica, en contraposición con otros países como Brasil que aconteció a principios de la década del 70 (SAMPAIO, 2005) y Chile que data del año 1981 (CRUZ, 2011). En este sentido, el estudio del aprendizaje y su profesionalización revistió características más controvertidas y polémicas en el contexto argentino.

Una evidencia empírica de ello es la diversidad de criterios en torno a la inserción universitaria de la Psicopedagogía que se observa hasta la actualidad. En ciertas universidades se constituye como una oferta de grado (Universidad Nacional del Comahue, Universidad Nacional de La Rioja, Universidad Nacional de Río Cuarto, Universidad Nacional de San Martín, Universidad Católica Argentina, Universidad John F. Kennedy, entre otras), mientras que en la Universidad de Buenos Aires desde 1986 es una asignatura de la carrera de Psicología y una de las cinco orientaciones de la carrera de Ciencias de la Educación (DI SCALA; PEREYRA, 2001; KAROL, 1992; MÜLLER, 2000).

Existen dos publicaciones que demuestran que los primeros debates y discusiones científicas en la comunidad académica se instalaron a fines de la década del 80. En primer lugar, se halló el cuestionamiento epistemológico de Castorina (1989) en el capítulo "Los obstáculos epistemológicos en la constitución de la Psicopedagogía" que forma parte de una compilación más amplia dedicada a los problemas de la Psicología Genética. El autor postuló respecto a la Psicopedagogía: 
Su status es el de ser una intervención en el campo del aprendizaje, sea como prevención o como diagnóstico y tratamiento de trastornos, sea como modificación del proceso de aprendizaje escolar (...) De momento, tal "disciplina" es un conjunto de prácticas dirigidas al campo del aprendizaje que reclama una fundamentación teórica más rigurosa ( $p$. 216).

En segundo lugar, Levy (1990) en "Sujeto y objeto en la clínica psicopedagógica. Su presencia y posición" problematizó la especificidad de la función terapéutica del psicopedagogo circunscripta en una práctica clínica interdisciplinar.

A nivel teórico-metodológico, esta condición interdisciplinar implicó la complementación de distintas teorías, técnicas y prácticas; algunas de ellas compatibles y otras, en franca contradicción (ARIAS, 2007; ARTACHO; VENTURA, 2011). A nivel profesional, el quehacer psicopedagógico se especializó en el diagnostico, tratamiento y pronóstico de los problemas de aprendizaje implicados en contextos educativos formales (BARTOLINI, 2004; NAZUTTI, 2009).

Estudios actuales demostraron que la formación universitaria y los perfiles profesionales conservan la mayor parte de estas características (HERNÁNDEZ; DE LOS SANTOS, 1998; MATTEODA, 1998; VENTURA; BORGOBELLO; PERALTA, 2010).

A pesar de que la Psicopedagogía es considerada como un campo en construcción (CABRERA PÉREZ; BETHENCOURT BENÍTEZ, 2010; CANTÚ; DIÉGUEZ, 2008; ORTIZ TORRES, 2000), escasas investigaciones han estudiado la génesis del campo conceptual y profesional psicopedagógico.

En este marco, cabe señalar que se encuentran en redefinición constante planteos como ¿por qué la Psicopedagogía adquirió autonomía académica antes de consolidarse como disciplina?; ¿por qué se creó la primera carrera de Psicopedagogía en Argentina?; ¿por qué surge en 1956 como una carrera de grado legítima sólo en el ámbito académico privado?; entre otros.

Es preciso mencionar que un estudio en profundidad de estos aspectos excede ampliamente los alcances del presente artículo. Es decir, aquí el propósito no es responder de manera concluyente a estos interrogantes, sino contextualizar y relacionar hechos significativos que permitan reflexionar y comprender, de manera preliminar, las controversias actuales en torno a este tema.

En este sentido, se considera que "para comprender el estado actual del campo psicopedagógico es necesario entenderlo como producto de un proceso histórico desarrollado en el marco de determinadas condiciones sociales y científicas" (BARAVALLE, 1995, p. 20).

El objetivo de este trabajo es explorar los modos en que se configuró el campo psicopedagógico en Argentina y los principales antecedentes que favorecieron la aparición de la práctica psicopedagógica durante 
la primera mitad del siglo XX y que, asimismo, posibilitaron su institucionalización académica en 1956.

De aquí que para analizar el origen de la Psicopedagogía es necesario situarlo en un contexto de tradiciones conceptuales psicológicas y educativas en torno a una nueva problemática social: el fracaso escolar. Para ello, se realizará una revisión de los acontecimientos sociales e históricos más significativos que operaron en la estructuración del campo psicopedagógico y en su inserción en el ámbito académico.

\section{Configuraciones socio-históricas del campo psicopedagógico}

A principios del $\mathrm{XX}$, el desarrollo y la producción científica argentina recibió la impronta de corrientes teóricas europeas (BRIGNARDELLO, 1983). Entre las principales influencias se destacó la Psicología positivo-experimental, entrelazada fuertemente a la Pedagogía y la Psiquiatría preocupada por la asistencia infantil desde criterios de normalidad-anormalidad (LUS, 2005).

En este marco, el retraso mental se constituyó como una categoría diagnóstica que indicaba afecciones físicas y mentales que reclamaban estrategias de reeducación y reinserción "plena" de los individuos a la sociedad (CABRERA PÉREZ; BETHENCOURT BENÍTEZ, 2010).

De aquí que en España los primeros trazos psicopedagógicos se lograron a partir de la "convergencia del biologismo, el positivismo y la psicología experimental en torno a ese lugar fronterizo de la educación que persiguió (...) la definición de una nueva pedagogía" (MOREU; BISQUERRA, 2003, p. 19). Em Francia, la primera aparición del término Psicopedagogía se produjo en el ámbito francófono (Francia, Bélgica y Suiza -especialmente la Escuela de Ginebra-) en el año 1908, en el marco de una conceptualización de "Paidotécnia". Es decir, vinculando los estudios psiquiátricos infantiles y las investigaciones psicométricas.

Paralelamente, se introdujo socialmente la representación de la infancia como un proceso característico y peculiar en el desarrollo de los seres humanos (VAN DER HORST, 1999). De aquí que la niñez se distinguió evolutivamente de la adultez.

Asimismo, el escenario argentino transitaba por épocas socialmente productivas $y$, al mismo tiempo, convulsionadas debido esencialmente a las oleadas migratorias y a las políticas modernas de constitución del Estado-Nación (BRÍGIDO, 2004; JARDÓN; FERNÁNDEZ, 2010; TENTI FANFANI, 2001).

A los fines de favorecer el proyecto de unidad estatal, se le asignó a la educación una función política. El surgimiento del Sistema Educativo, de carácter obligatorio y gratuito, estuvo encargado de 
"disciplinar a las masas nativas e inmigrantes en los nuevos patrones políticos, ideológicos y culturales" (DE MIGUEL, 1999, p. 178) e incluirlos en un nuevo orden social.

Según Tedesco, (2003) la universalización de la enseñanza tuvo lugar solamente en el nivel primario, mientras que la escuela media y la superior estaban reservadas para un sector oligárquico, es decir, una minoría nativa o elite. Este sistema tuvo como modelo el paradigma educativo inglés. El contenido de la educación oligárquica era enciclopédico con predominio de las materias humanísticas, propias de la enseñanza tradicional.

Los medios de control social comprendieron desde la determinación de un currículum hasta la selección el personal y desde la difusión de ciertos valores hasta impedir que ciertos sectores de la población accedieran a determinadas esferas del conocimiento.

En este sentido, la propuesta escolar era normalizadora dado que tendía a la adaptación a determinados patrones con la finalidad de homogeneizar al educando, reflejadas en la conformación de los sucesivos currículos de las escuelas normales (GUTIÉRREZ, 2007).

El Normalismo defendió la posibilidad de educar efectivamente al sujeto, la primacía de la ciencia, el orden, el progreso y la necesidad de criterios científicos de demarcaran los métodos correctos para la enseñanza. Se caracterizó además por proponer una minuciosa reglamentación de la actividad que contemplara hasta los más mínimos detalles de comportamiento, ejercitación motriz y mobiliario adecuado (TEDESCO, 2003).

El liderazgo del Normalismo significó a nivel popular un giro sin precedentes. En la población nativa, exigió la inserción de grupos ágrafos en una lógica simbólica convencional inherente a la cultura letrada. En la población inmigrante, implicó aprender una lengua e integrarse a una nueva cultura (DE MIGUEL, 1999; DE MIGUEL; DE BIAGGI; ENRICO; ROMAN, 2007).

En este marco, el aprendizaje se concibió como un producto tributario de una herencia genética cuyas capacidades podían ser medidas y normalizadas a través de la observación y reeducación sensorial. EI alumno tenía una figura pasiva y receptiva que aprendía mediante el refuerzo de habilidades cognitivas como lectura, memorización lineal y aplicación de saberes (JARDÓN; FERNÁNDEZ, 2010).

Por tanto, constituido el Sistema Educativo, aparece la posibilidad de "fracaso escolar" (ASCOLANI; NICOLAU, B.; TALVO; NICOLAU, M.; NAZUTTI; CEBALLOS; RUGGERONI; LÓPEZ, 2007) cuyo tratamiento fue abordado inicialmente por la práctica cotidiana y espontánea de las maestras particulares (BARAVALLE, 1995). La prevención y detección de las dificultades cumplía una función de control social (CASTILLO; ROJ AS BREU, 2008).

En suma, la conformación del Estado-Nación, el surgimiento del Sistema Educativo, la aparición de la categoría de "fracaso escolar" y 
la intervención de las maestras recuperadoras sentaron las bases para la configuración del campo psicopedagógico.

\section{Precursores y antecedentes institucionales de la Psicopedagogía}

En el escenario académico argentino, Gottheld (1969) consideró a Mercante como uno de los principales precursores de la psicopedagogía positivista, enarbolando los principios de Darwin, Krause y Comte. En 1981, Mercante fundó el Primer Laboratorio de Psicología Experimental en San Juan cuyas líneas de investigación fueron el estudio de la disortografía, la lectura mecánica, la imaginación reproductora y los métodos efectivos de enseñanza. Asimismo, fundó y dirigió una revista abocada a la investigación psicopedagógica, "Archivos de Pedagogía y Ciencias Afines" (J ARDÓN; FERNÁNDEZ, 2010).

En Mendoza, Leónidas Aguirre propuso que la Pedagogía debía obtener sus fundamentos en la Psicología, cuya enseñanza en los Colegios Nacionales, era básicamente de corte experimental. Así fue dispuesto en un Decreto Nacional promulgado en el año 1904 bajo la dirección del Ministro de Educación Joaquín V. González (GOTTHELD, 1969).

En la Universidad de La Plata, la enseñanza de la Psicología comenzó a funcionar en 1905 en el marco de una sección pedagógica organizada en la Facultad de Ciencias Jurídicas y Sociales. En 1914, esta sección se designó a la Facultad Ciencias de la Educación. Este plan de estudios estaba destinado a la formación de docentes y la asignatura "Psicopedagogía" se dictaba en el segundo año, junto con Psicología Experimental y Metodología.

En 1920, dicha asignatura comenzó a estar a cargo de Rodolfo Senet. El mismo fue un exponente del asociacionismo que sistematizó aportes de la Psicología infanto-juvenil de la época, muestra de ello son "Psicología de la adolescencia, la pubertad y la juventud" (GOTTHELD, 1969) y "Nota sobre los retrasados escolares" (CASTILLO; ROJ AS BREU, 2008).

En este sentido, las primeras alusiones y usos del término "Psicopedagogía" estuvieron íntimamente vinculados a la aplicación del saber psicológico en ámbitos formales o reglados de enseñanzaaprendizaje.

A partir de la década del 20, comenzó a perfilarse la orientación psicotécnica marcada por una tendencia técnica-profesional. La emergencia de posturas más humanistas guardaron una estrecha vinculación con la "inestabilidad política, golpes militares, debates en el eje idealismo-materialismo, cambios en la filosofía oficial" (ALONSO, 1999, p. 4). 
La Psicotécnia, heredera de la revolución industrial y modelo de trabajo fordista, se constituyó como un abordaje aplicado en el cual confluyeron la Medicina, la Psicología, la Economía y la Estadística. Las áreas más desarrolladas de este enfoque fueron la evaluación y organización de educativa en grados de acuerdo a sus capacidades mentales, el diagnóstico de retardos escolares y la prescripción de tratamientos médico-pedagógicos y la orientación vocacional-laboral (LUQUE, 2009a).

Representantes de este periodo resultaron el Instituto de Psicotecnia y Orientación Laboral en 1925, el Instituto de Psicología Correctiva y Escuela de Niños Débiles del Consejo Nacional de Educación en 1928, la Escuela de Psicología Correctiva en 1929 (ALONSO, 1999; ROSSI, 2008). De aquí que se privilegió una concepción del aprendizaje en términos funcionales, revistiéndose como una herramienta para el progreso social. Durante este periodo, el problema de aprendizaje comenzó a representar el fracaso en la vida ( $\mathrm{RICCl}, 2004)$.

Años más tarde, la corriente psicotécnica se sistematizó a partir de la implementación de la Licenciatura de Psicotecnia y Orientación Profesional, dividida en 5 años de formación (LUQUE, 2008b). En 1951, se organizó el Gabinete de Psicotecnia de Paraná. El 29 de abril de 1953 se fundó la carrera de Auxiliar en Psicotécnia en la Facultad de Filosofía, Letras y Ciencias de la Educación de la Universidad Nacional del Litoral con sede en Rosario con los siguientes fines (LUQUE, 2008b, p. 84):

\begin{abstract}
De los considerandos (...) destaquemos el designio de formar personal especializado para construir un conocimiento sistemático, con base científica, de los alumnos y propiciar el mejoramiento de los fines educativos (...) Asimismo, la carrera debía impulsar la investigación educativa y se destinó al perfeccionamiento de los egresados de las escuelas normales (maestros).
\end{abstract}

No obstante, en 1955 la Universidad Nacional del Rosario resolvió el cierre de la carrera Auxiliar en Psicotecnia y su reemplazo para el siguiente año académico por la carrera de Psicología (LUQUE, 2008b). Sin embargo, es posible plantear que se generó un área de vacancia profesional que intentó ser cubierta por la carrera de Psicopedagogía. Un antecedente que puede indicar la vinculación entre la psicotecnia y psicopedagogía es la creación del Instituto de Investigaciones Psicopedagógicas fundado en 1948 por Plácido Horas en la Universidad de Cuyo - con sede en San Luis-. A partir del año 1952 el Instituto editó la Revista Anales del Instituto de Investigaciones Psicopedagógicas. Muñoz consideró que aquí se desplegó y enraizó el campo de la orientación vocacional abrigando las concepciones psicotécnicas vinculadas a la orientación profesional (MUÑOZ, 2011). En suma, si bien las primeras referencias institucionales de lo 
psicopedagógico datan de las primeras décadas del siglo $\mathrm{XX}$, los primeros esbozos de sistematización de este campo puede vislumbrarse mediante el advenimiento de la Psicotecnia en el escenario universitario.

\title{
4 Institucionalización académica de la Psicopedagogía
}

El enfoque psicopedagógico se imbricó en un nuevo ámbito profesional delineado por tres áreas indisolublemente ligadas: la intervención, la orientación y la educación (SOLÉ, 1998). Es en este contexto que se organizó la carrera de Psicopedagogía en la Universidad del Salvador para brindar bases científicas a la práctica espontánea de las maestras particulares que se ocupaban de un nuevo objeto de intervención, el fracaso escolar.

El siguiente fragmento comprende la naturaleza de los fundamentos que predominaron en la dinámica de estructuración del campo psicopedagógico:

\begin{abstract}
Al crearse la carrera de Psicología, en la recién fundada Universidad del Salvador, surgió la inquietud de abrir una rama de esta disciplina dedicada explícitamente al perfeccionamiento docente y al ámbito educativo. De ahí nació la Psicopedagogía como carrera universitaria de tres años (MÜLLER, 2000, p. 1).
\end{abstract}

En otros términos, la Psicopedagogía se configuró como una rama aplicada de la Psicología, destinada básicamente al perfeccionamiento docente y con un recorte dirigido explícitamente a los problemas de aprendizaje originados en el Sistema Educativo.

Se considera que una revisión de los principales representantes de la Psicopedagogía permite vislumbrar los modelos teóricos que sustentaron y orientaron las incipientes prácticas psicopedagógicas. Cabe destacar que la reseña que se presenta a continuación no es exhaustiva dado que el énfasis se ubicó en mencionar ciertas producciones científicas que lograron una integración conceptual específica, estructuraron de manera significativa el campo psicopedagógico.

Durante la década del 60 , no se relevaron referentes teóricos propios. En este periodo, la función del psicopedagogo estuvo solapada bajo la figura del médico, que legitimó su práctica de rehabilitación escolar prescribiendo el plan de trabajo de acuerdo con la afección del alumno (BARAVALLE, 1995). En este sentido, la formación universitaria se basó en un modelo reeducativo (LUS, 2005).

A principios de los años 70, Sara Paín se convirtió en una pionera de la Psicopedagogía argentina, articulando los aportes de la Psicología Genética, la Psicometría y el Psicoanálisis para abordar los problemas 
de aprendizaje. Entre sus obras se destacan Psicometría genética (1971), Diagnóstico y tratamiento de los problemas de aprendizaje (1983) y La génesis del inconsciente (1984). Es posible inferir que se produjo un giro conceptual en la formación del psicopedagogo, la formación comenzó a caracterizarse como una psicoterapia escolar (PAín, 2003).

Es probable que este movimiento haya influido en la emergencia de los debates teórico-epistemológicos iniciales en la década del 80 (CASTORINA, 1989; LEVY, 1990) dado que se desdibujó la imagen del psicopedagogo como un asistente reeducativo del maestro, aproximándose a la figura terapéutica del psicólogo.

Por su parte, Marina Müller fundó la primera revista de Psicopedagogía con una visión integral y humanista denominada hasta la actualidad Aprendizaje Hoy. Revista de Actualidad Psicopedagógica. Otras producciones de la autora son "Aprender para ser: principios de la psicopedagogía clínica" (1994), "Docentes tutores. Orientación educativa y tutoría" (1998), "Formación docente y psicopedagógica: estrategias y propuestas para la intervención educativa" (2008).

Alicia Fernández recuperó los aportes de Paín situando el foco de su trabajo en la teoría psicoanalítica y forjando una lectura psicopedagógica. Resultan expresiones de ello, La inteligencia atrapada. Abordaje psicopedagógico clínico del niño y su familia (1987), "Poner en juego el saber" (2000), "Los idiomas del aprendiente" (2000), "Psicopedagogía en psicodrama" (2009).

En el marco de la Epistemología Convergente, Jorge Visca desarrolló numerosas producciones psicopedagógicas recibiendo las contribuciones de la Psicología Genética, la Psicología Social de Pichón-Riviere y el Psicoanálisis. Una de sus propuestas originales consistió en la creación de técnicas proyectivas, las cuales se encuentran incluidas en el libro que lleva su nombre Técnicas proyectivas psicopedagógicas (1994).

Cabe mencionar que los aportes de Alicia Fernández y Jorge Visca han trascendido las fronteras nacionales, impactando especialmente en el ámbito psicopedagógico brasilero.

En base a revisión efectuada, es posible suponer que desde su inserción universitaria la Psicopedagogía se constituyó como un campo de prácticas institucionalizadas para resolver los problemas de aprendizaje escolares. De aquí que sus modelos de formación universitaria se sustentaron sobre la articulación de distintos marcos teóricos fundamentalmente psicológicos.

\section{Consideraciones finales}

En el contexto sudamericano, la institucionalización académica de la 
Psicopedagogía se forjó en Argentina, sin embargo, existen escasas reseñas nacionales que revisen e identifiquen los procesos sociales y movimientos históricos que dieron lugar a la síntesis del discurso psicopedagógico. La historia española de la Psicopedagogía comparte características semejantes (MOREU; BISQUERRA, 2003).

Este artículo permitió entrever los modos en que se configuró el campo psicopedagógico en Argentina y los principales antecedentes que propiciaron su institucionalización académica en 1956 en la Universidad del Salvador (Buenos Aires). En este sentido, el surgimiento del Estado-Nación y el Sistema Educativo, la aparición del "fracaso escolar" abordado por la práctica de las maestras particulares se constituyeron como algunas de las condiciones necesarias para la conformación del campo psicopedagógico.

Asimismo, la necesidad de dotar de bases científicas y perfeccionar la intervención docente puede reflejarse en la sistematización de la carrera de "Psicotecnia" en el ámbito universitario. A posteriori de su clausura y surgimiento de la carrera de Psicología, se produce la inserción de la Psicopedagogía en un contexto académico privadoconfesional.

Siguiendo a Bravo (2009), la Psicopedagogía "partió del fenómeno educativo mismo de sus necesidades en el aula, considerándose una disciplina aplicada que utiliza los aportes de la psicología para enriquecer el quehacer educacional" (p. 219).

A través de los diversos modelos de formación universitaria de la carrera de Psicopedagogía, es posible observar la naturaleza aplicada de las prácticas psicopedagógicas dirigidas hacia la resolución de los problemas de aprendizaje escolares. Entre sus referentes teóricos se destacan: Sara Paín, Marina Müller, Alicia Fernández y Jorge Visca.

Tal como se mencionó previamente, este artículo se limitó a explorar aspectos sociales e históricos significativos que permitieran pensar la génesis y desarrollo del campo conceptual y profesional psicopedagógico. Por lo tanto, cabe señalar que resultan necesarios estudios posteriores con alcances descriptivos e interpretativos que permitan analizar con mayor profundidad esta temática.

Se considera se suma relevancia continuar con este tipo de estudios que permitan profundizar, contextualizar y comprender las raíces históricas y socio-culturales de las controversias y debates actuales que se sitúan entre las disciplinas implicadas en el campo del aprendizaje y, asimismo, al interior de la Psicopedagogía.

En esta dirección, se recupera el pensamiento de Ibáñez (1995) permitiendo ensayar futuras propuestas o líneas de investigación que problematicen aspectos como: ¿qué fundamentos metateóricos promovieron la institucionalización académica de la Psicopedagogía?; ¿qué condiciones generaron que el aprendizaje se formalice como un objeto de intervención que no podía ser explicado por la psicología o por la educación?; ¿qué concepciones de aprendizaje sustentaron 
estos desarrollos? En definitiva, repensar el campo de estudio del aprendizaje es revisar la historia de la psicopedagogía.

\section{Referencias}

ALONSO, M. Psicología en Argentina. In: ALONSO, M.; EAGLY, E. (Eds.). Psicología en las Américas. Caracas: SIP, 1999, p. 3-17.

ARIAS, P. La Psicopedagogía: sus vinculaciones históricas con otros saberes y su posición actual. Un debate necesario. Aprendizaje Hoy, n .67, p. 55-64, 2007.

ARTACHO, J. A.; VENTURA, A. C. Psicopedagogía e interdisciplina: reflexiones desde una perspectiva epistemológica. Aprendizaje Hoy, n. 80, p. 7-16, 2011.

ASCOLANI, A.; NICOLAU, B.; TALVO, B.; NICOLAU, M.; NAZUTTI, M.; CEBALLOS, J.; RUGGERONI, C.; LÓPEZ, M. La constitución del campo disciplinar de la Psicopedagogía en la Argentina. Manuscrito no publicado. Rosario: Universidad Nacional de San Martín - Unidad Académica Rosario, 2007.

BARAVALLE, L. La formación del campo profesional psicopedagógico: fundamentos teóricos y perspectivas como campo disciplinar. Jornadas Latinoamericanas, Estudios Sociales de la Ciencia y la Tecnología, Universidad Nacional de Quilmes, p. 18-20, 1995.

BARTOLINI, A. Relaciones entre formación e inserción laboral de los psicopedagogos en Entre Ríos. Una aproximación evaluativa. Ciencia, Docencia y Tecnología, v. 15, n. 29, p. 65-92, 2004.

BRAVO, L. Psicología educacional, Psicopedagogía y Educación especial. Revista II PSI, Facultad de Psicología, UNMSM, v. 12, n. 2, p. 217-225.

BRÍGIDO, A. El sistema educativo argentino. Elementos conceptuales, metodológicos y empíricos para su análisis. Córdoba: Bujas, 2004.

BRIGNARDELLO, L. Acerca de la Psicología Social en la Argentina. Revista Latinoamericana de Psicología, v. 15, n. 3, p. 397-401, 1983.

CABRERA PÉREZ, L.; BETHENCOURT BENÍTEZ, T. La psicopedagogía como ámbito científico-profesional. Electronic Journal of Research in Educational Psychology, v. 8, n. 2, p. 893-914, 2010.

CANTÚ, G.; DIÉGUEZ, A. Acerca de las investigaciones en Psicopedagogía clínica: algunos supuestos filosóficos. Perspectivas en Psicología, v. 5, n. 1, p. 9-15, 2008.

CASTILLO, C.; ROJAS BREU, G. La presencia del discurso psicológico en publicaciones específicas referidas a la educación: La Obra y EI Monitor de la Educación Común (1920-1935). Revista de Historia de la Psicología en Argentina, n. 1, p. 192-204, 2008.

CASTORINA, J. Los obstáculos epistemológicos en la constitución de 
la Psicopedagogía. In: CASTORINA, J.; PALAU, G.; AISENBERG, B.; DIBAR UCRE, C.; COLINVAUX, D. (Comps.). Problemas en Psicología Genética. Buenos Aires: Miño y Dávila, 1989, pp. 215232.

CRUZ, Y. ¿Qué es la psicopedagogía? Disponible en: <http://psicoeducativo.wordpress.com/2008/05/19/\% C2\% BF-quees-la-psicopedagogia/>. Extraído el: 15 Feb. 2011.

DE MIGUEL, A. Hegemonía y clausura del discurso normalista. In: ASCOLANI, A. (Comp.). La educación en Argentina. Estudios de historia. Rosario: Ediciones del Arca, 1999, p. 177-184.

DE MIGUEL, A.; DE BIAGGI, M.; ENRICO, J.; ROMAN, M. Normalismo, cultura letrada y resistencia de la oralidad en la historia de la lectura y la escritura en Argentina. Ciencia, Docencia y Tecnología, n. 34, p. 97-127, 2007.

DI SCALA, M.; PEREIRA, M. Psicopedagogía. Interrogantes sobre su estatuto como disciplina. Manuscrito no publicado. Buenos Aires: Ficha del Departamento de Publicaciones de la Facultad de Psicología, Universidad de Buenos Aires, 2001.

FERNÁNDEZ, A. La inteligencia atrapada. Abordaje psicopedagógico clínico del niño y su familia. Buenos Aires: Nueva Visión, 1987.

$\overline{2} \overline{0} \overline{0}$.

Los idiomas del aprendiente. Buenos Aires: Nueva Visión,

$\overline{2} \overline{0} \overline{0}$.

Visión, 2009.

Poner en juego el saber. Buenos Aires: Nueva Visión,

Psicopedagogía en psicodrama. Buenos Aires: Nueva GARCÍA, J-N. Las dificultades de aprendizaje y otros trastornos del desarrollo. eduPsykhé, v. 1, n. 2, p. 295-312, 2002.

GOTTHELD, R. Historia de la psicología en la Argentina (II parte). Revista Latinoamericana de Psicología, v. 1, n. 2-3, p. 183-198, 1969.

GUTIERREZ, T. Políticas de orientación agrícola y pedagogía normalista. Entre Ríos, Argentina, 1900-1920. Perfiles educativos, v. 29, n. 117, p. 85-110, 2007.

HENAO LÓPEZ, G.; RAMÍ REZ NIETO, A; RAMÍ REZ PALACIO, C. Qué es la intervención psicopedagógica: definición, principios y componentes. AGO.USB, v. 6, n. 2, p. 215-228, 2006.

HERNÁNDEZ, L.; DE LOS SANTOS, M. La cientificidad del quehacer psicopedagógico. Aprendizaje Hoy, n. 39, p. 41-44, 1998.

IBAÑEZ, T. La tensión esencial de la psicología social. In: PAÉZ, D.; VALENCIA, J.; MORALES, J.; SARABIA, B.; URSUA, N. (Eds.). Teoría y método en psicología social. Barcelona: Antrophos, 1995, p. 1328.

JARDÓN, M.; FERNÁNDEZ, V. Análisis de un estudio cuantitativo sobre los problemas de aprendizaje en registros formales de 
documentación (1910). Revista Historia de la Psicología en Argentina, n. 3, p. 143-150, 2010.

KAROL, M. Algunas cuestiones para pensar acerca de la psicopedagogía y su constitución como disciplina. Manuscrito no publicado. Buenos Aires: Ficha del Departamento de Publicaciones de la Facultad de Psicología, Universidad de Buenos Aires, 1992.

LEVY, E. Sujeto y objeto en la clínica psicopedagógica. Su presencia y posición. Temas de Psicopedagogía, n. 5, p. 12-18, 1989.

LUQUE, E. Algunas instituciones de orientación profesional vinculadas a la psicotecnia en Buenos Aires (década del 30). Revista de Historia de la Psicología en Argentina, n. 2, p. 71-81, 2009a.

LUQUE, E. La transmisión académica de la psicotecnia en Rosario y Tucumán (1950-1957). Revista de Historia de la Psicología en Argentina, n. 2, p. 82-92, 2009b.

LUS, M. De la integración escolar a la escuela integradora. Buenos Aires: Paidós, 2005.

MATTEODA, M. Consideraciones acerca de la práctica, la formación y la investigación psicopedagógica. Contextos de Educación, v. 1, n. 1, p. 112- 121, 1998.

MOREU, A.; BISQUERRA, R. Los orígenes de la Psicopedagogía: el concepto y el término. Revista Española de Orientación y Psicopedagogía, v. 13, n. 1, p. 17-29, 2002.

MÜLLER, M. Aprender para ser: principios de psicopedagogía clínica. Buenos Aires: Bonum, 1994.

MÜLLER, M. Docentes tutores: orientación educativa y tutoría. Buenos Aires: Bonum, 1998.

MÜLLER, M. Perspectivas de la Psicopedagogía en el comienzo del milenio. Publicación virtual de la Facultad de Psicología y Psicopedagogía de la USAL, v. 1, n. 2, p. 1-10, 2000.

MÜLLER, M. Formación docente y psicopedagógica: estrategias y propuestas para la intervención educativa. Buenos Aires: Bonum, 2008.

MUÑOZ, M. Investigación y política en los Anales del Instituto de Investigaciones Psico-Pedagógicas de la Universidad Nacional de Cuyo 1948-1966. Constitución del campo de la orientación vocacional. Diálogos. Revista Científica de Psicología, Ciencias Sociales, Humanidades y Ciencias de la Salud, v. 2, n. 1, p. 49-66, 2001.

NAZUTTI, M. El quehacer psicopedagógico en sus orígenes. Revista del Colegio de Psicopedagogos, v. 1, n. 2, p. 3-4, 2009.

ORTIZ TORRES, E. El peligro del eclecticismo en las investigaciones psicopedagógicas contemporáneas. El caso de las concepciones de Vigotsky y Piaget. Revista Pedagogía Universitaria, v. 5, n. 3, p. 14-24, 2000.

PAI N, S. Psicometría genética. Buenos Aires: Nueva Visión, 1971.

PAIN, S. Diagnóstico y tratamiento de los problemas de 
aprendizaje. Buenos Aires: Nueva Visión, 1983.

PAIN, S. La génesis del inconsciente. Buenos Aires: Nueva Visión, 1984.

PAÍ N, S. La Psicopedagogía en la actualidad. In: LAINO, D.; PAÍN, S.; AGENO, R. (Comps.). La Psicopedagogía en la actualidad. Nuevos aportes para una clínica del aprender. Rosario: Homo Sapiens, 2003, p. 23-32.

$\mathrm{RICCl}, \mathrm{C}$. Psicopedagogía: Aportes para una reflexión epistemológica. Revista Aprendizaje Hoy, n. 57, p. 7-20, 2004.

ROSSI, L. Telma Reca. Revista de Historia de la Psicología en Argentina, n. 1, p. 185-191, 2008.

SAMPAIO, S. Um pouco da história da psicopedagogía, 2005. Disponible en: <http://www. psicopedagogia.com. br/opiniao/opiniao. asp?entrlD=422 >. Extraído el: 15 Feb. 2011.

SOLÉ, I. Orientación educativa e intervención psicopedagógica. Barcelona: I.C.E.-Horsori, 1998.

TEDESCO, J. Educación y sociedad en la Argentina (18801945). Buenos Aires: Siglo XXI, 2003.

TELLO, J. EI Prácticum en la Licenciatura de Psicopedagogía en la Universidad de Huelva. Implicaciones en la formación del Psicopedagogo e incidencia en la apertura de yacimientos de empleos. 2009. Tesis (Doctorado en Educación) - Departamento en Educación, Universidad de Huelva, España.

TENTI FANFANI, E. Sociología de la Educación. Buenos Aires: Universidad Nacional de Quilmes, 2001.

VAN DER HORST, C. La escuela normal. Una institución para el orden. In: ASCOLANI, A. (Comp.). La educación en Argentina. Estudios de historia. Rosario: Ediciones del Arca, 1999, p. 129-144.

VENTURA, A.; BORGOBELLO, A.; PERALTA, N. Representaciones acerca del campo disciplinar y formación profesional de la psicopedagogía en un contexto académico argentino. Revista Española de Orientación y Psicopedagogía, v. 21, n. 3, p. 653668, 2010.

VISCA, J. Técnicas proyectivas psicopedagógicas. Buenos Aires: Visca \& Visca, 1994.

Endereço para correspondência

Ana Clara Ventura

Instituto Rosario de Investigaciones en Ciencias de la Educación-IRICE (CONICET/UNR). Dirección postal: 27 de Febrero 210 bis (Rosario, Santa Fe, Argentina). Código postal: 2000.

Endereço eletrônico: ventura@irice-conicet.gov.ar

Raúl Gagliardi

Instituto Rosario de Investigaciones en Ciencias de la Educación-IRICE (CONICET/UNR). Dirección postal: 27 de Febrero 210 bis (Rosario, Santa Fe, Argentina). Código postal: 2000. 
Endereço eletrônico: gagliardi@irice-conicet.gov.ar

Nora Moscoloni

Instituto Rosario de Investigaciones en Ciencias de la Educación-IRICE

(CONICET/UNR). Dirección postal: 27 de Febrero 210 bis (Rosario, Santa

Fe, Argentina). Código postal: 2000.

Endereço eletrônico: moscoloni@irice-conicet.gov.ar

Recebido em: 26/09/2011

Reformulado em: 04/04/2012

Aceito para publicação em: 25/05/2012

Acompanhamento do processo editorial: Ana Maria Jacó Vilela

\section{Notas}

* Licenciada en Psicopedagogía. Profesora Universitaria en Psicopedagogía. Doctoranda en Psicología.

**Doctor en Ciencias (Biología). Doctor en Educación por la Universidad de Ginebra (Suiza). Investigador Independiente de CONICET. Director del Instituto Rosario de Investigación en Ciencias de la Educación (IRICE-CONICET-UNR).

$* * *$ Doctora por la Universidad Nacional de Rosario (Argentina). Profesional Principal de CONICET. 